\title{
Metodología de enseñanza para la producción textual efectiva en alumnos de primer semestre de Bachillerato. (Centro de Bachillerato Tecnológico Industrial y de Servicios No. 11 (CBTIS), Hermosillo, Sonora, México)
}

\author{
Teaching Methodology for Effective Text Production to First \\ Semester College Students (Technological, Industrial, and Services \\ High School No. 11 [CBTIS] in Hermosillo Sonora)
}

\begin{abstract}
ALma Judith NúñEz Soto, Universidad de Sonora, Sonora, México
\end{abstract}
\author{
Volumen 1, Número 1 \\ Edición especial. Febrero de 2016
}

p. $118-143$

Este número se publicó el 5 de febrero de 2016

Artículo recibido: 20 de septiembre de 2014

Artículo aprobado: 10 de noviembre de 2015

ISSN: 2448-5942, doi: https://doi.org/10.36799/el.v1i1.25

\section{Citar este artículo como:}

Núñez Soto, A. J. (2016). Metodología de enseñanza para la producción textual efectiva en alumnos de primer semestre de Bachillerato. (Centro de Bachillerato Tecnológico Industrial y de Servicios No. 11 (CBTIS), Hermosillo, Sonora, México). Estudios גambda. Teoría y práctica de la didáctica en lengua y literatura, 1(1), 118-143. https://doi.org/10.36799/el.v1i1.25

Derechos de autor: El autor o autores conservan en todo momento sus derechos morales y patrimoniales sobre la obra; la obra no se puede alterar, transformar o ampliar; siempre debe reconocerse la autoría del documento

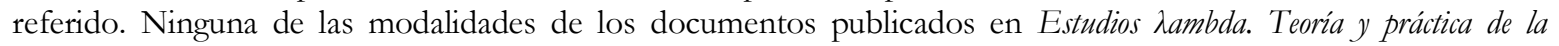
didáctica en lengua y literatura tienen fines comerciales de naturaleza alguna.

Los contenidos de este artículo están bajo la licencia de Creative Commons Atribución No Comercial- Sin Derivadas 4.0 Internacional @⿶凵 


\title{
Metodología de enseñanza para la producción textual efectiva en alumnos de primer semestre de Bachillerato. (Centro de Bachillerato Tecnológico Industrial y de Servicios No. 11 (CBTIS), Hermosillo, Sonora, México)
}

\author{
Teaching Methodology for Effective Text Production to First Semester College \\ Students (Technological, Industrial, and Services High School No. 11 \\ [CBTIS] in Hermosillo Sonora)
}

ALMA JUDITH NúÑEZ SOTO ${ }^{1}$

\begin{abstract}
RESUMEN
Este trabajo expone los resultados de una investigación-acción, específicamente, un estudio de caso inductivo de escritura enfocado en las estructuras básicas de la lengua, cuyo objetivo primordial se basa en la metodología que se implementa en la enseñanza de la escritura en alumnos de primer semestre del CBTis11, utilizando una muestra de diez estudiantes al azar y abarcando desde la oración simple, los diferentes tipos de párrafos hasta la estructura textual. Utilizando la exploración y descripción de párrafos, tipos y conectivos. El referente primordial de dicho estudio es María Teresa Serafini, quien considera que escribir está relacionado con una serie de operaciones fundamentales: Planeación, textualización y revisión: "Reunir y organizar las propias ideas, escribir esquemas, asociar cada idea, desarrollar los razonamientos, revisar el propio escrito" (1997). Propone tres fases: Preescritura, escritura y postescritura. La primera reúne la información y la manipula antes del desarrollo; la segunda construye lingüísticamente el texto y la última revisa la producción escrita. La investigación se basó en dos escritos propios del alumno: Un diagnóstico y un texto final los cuales arrojaron información distinta de coherencia estructural avanzada. Para obtener la información deseada se basó en categorías específicas de la lengua: Coherencia, cohesión y estructura del párrafo, dentro de ésta última, se aludió a la puntuación, sintaxis, conectores, léxico y aspectos gráficos. Los resultados obtenidos del texto final respecto al diagnóstico son los siguientes: Los alumnos lograron escribir párrafos complejos, utilizando conectores y puntuación adecuada, su acervo lingüístico aumentó, su coherencia y cohesión presentaron mejoría.
\end{abstract}

PALABRAS CLAVE: Oración, párrafo, coherencia, cohesión.

\begin{abstract}
The present paper presents the results of a research-action, specifically a case study of inductive writing, focusing on the basic structures of the language, whose primary objective is based on the methodology that is implemented in the teaching of writing in first semester students of CBTIS 11, using a sample of ten randomly selected students; ranging from simple sentences, different types of paragraphs up to the text structure, using exploration and description paragraphs, types and connectives. The primary reference for the study is originated from Maria Teresa Serafini who believes that writing is related to a number of key operations: planning, text construction and reviewing. "Collecting and organizing our own ideas, writing outlines, associating each idea, developing arguments, and reviewing the text itself "(1997). It proposes three phases: prewriting, writing and post writing. The first phase consists in gathering information and handling it before its development; the second consists of the linguistic construction; the latter reviews the written production. The research was based on two texts prepared by the student: a diagnosis and a final text which yielded information other than advanced structural coherence. To obtain the desired information, the work was based on specific categories of language: coherence, cohesion and paragraph structure, in this last category specifically punctuation, syntax, connectors, lexical and graphic aspects. The results obtained from the final text regarding diagnosis are: students were able to write complex paragraphs using connectors and proper punctuation, their linguistic knowledge increased; coherence and cohesion showed improvement.
\end{abstract} 1 Docente en el Departamento de Letras y Lingüística, Universidad de Sonora. Licenciado en Lingüística, Maestría en
Educación. almanunez@,capomo.uson.mx

Artículo recibido: 20 de septiembre de 2014

Artículo enviado a corrección: 24 de septiembre de 2015

Aprobado: 10 de noviembre de 2015

Núñez doi: https://doi.org/10.36799/el.v1i1.25 Volumen 1, Número 1, Año 2016, ISSN: 2448-5942 
KEYWORDS: Sentence, paragraphs, coherence, cohesion.

\section{INTRODUCCIÓN}

En la actualidad, la enseñanza de la lengua se ha enfocado en el hábito de la lectura y ha descuidado el de la escritura. Este paradigma no es novedoso, en Latinoamérica, por más de una década se han hecho investigaciones acerca del tema y se han dado diversas propuestas para solucionarlo. El interés de este estudio radicó en comprender los problemas que presentan los estudiantes en la escritura, conocer qué competencias utilizan y qué metodología emplea el docente para lograr la producción textual.

Es importante aclarar que muchas de las investigaciones realizadas por diversos docentes no han arrojado información relevante de manera completa, es decir, no se consideraron todos los aspectos que involucra nuestra lengua: gramática, sintaxis, léxico (semántica y pragmática); sólo han aportado información de ciertos aspectos.

La metodología de enseñanza utilizada en el proyecto estudiado es muy interesante, ya que involucró todas las categorías de nuestro idioma además de que proporcionó un vínculo importante entre la competencia lectora-escritora, con el conocimiento metalingǘstico de los estudiantes así como con la transversalidad presentada entre las materias.

El docente manejó un proceso que consta de cuatro etapas básicas: diagnóstico, propuesta, implementación y evaluación. La primera fue un escrito libre que se redactó al principio del semestre donde el alumno escribió de acuerdo a su competencia metalingüística basada en estructura, forma, extensión y organización textual. La segunda y tercera se originaron a partir de la propuesta de María Teresa Serafini, por lo que se manejó primeramente la enseñanza de la lectura como un proceso donde se realizan tres etapas: preescritura, escritura y revisión; seguidamente, se trabajó la escritura, donde se estructuran ocho tipos de párrafos: enumerativo, desarrollo de concepto, de enumeración, de secuencia, de causa-efecto, de problema-solución, comparativo, introducción y cierre. Es importante enfatizar que los alumnos debieron escribir párrafos continuamente utilizando tinta roja para señalar conectores y signos de puntuación, contar palabras dentro de cada tipo de oración principal y secundaria, considerando +/- 20 palabras para la oración y de 60 a 120 palabras para un párrafo. La cuarta y última etapa manejó la evaluación continua, es decir, la estructura de párrafos durante todas las semanas de impartición de clase; posteriormente la evaluación 
parcial, la cual abordó la escritura de párrafos vistos en la primera y segunda unidad; finalmente la evaluación final consistió en un escrito que el alumno redactó a partir de conocimientos previos adquiridos a través de diversas lecturas hechas a lo largo del semestre, en el escrito se utilizaron los ocho tipos de párrafos, respetando las rúbricas señaladas para su elaboración.

La relación lectura-escritura es inminente. Desde los primeros acercamientos con el texto se establece una relación no solamente visual, sino que involucra también esa seriación de procesos cognitivos que ayudan a decodificar la información presentada en el texto. Como se mencionó, el proyecto se basó principalmente en la propuesta de María de Teresa Serafini quien aborda la estructura proposicional por medio de tipología de párrafos.

El trabajo de investigación aquí expuesto es de tipo cuantitativo-descriptivo de interacción e introspección grupal institucional; es un estudio de caso, ya que se analizaron datos expresados en el lenguaje escrito, específicamente la estructura oracional de textos en alumnos de nivel medio superior; donde se exploraron y describieron tipos de párrafos, conectivos, así como otros aspectos que se encuentran implícitos dentro de la escritura. Representa la parte final de un proceso de análisis, observación, anotación, interpretación de documentos en el contexto escolar, el cual se llevó a cabo en el Centro de Bachillerato Tecnológico industrial y de servicio No. 11 (CBTis 11), en Hermosillo, Sonora.

La investigación de la producción de textos en el nivel medio superior ha sido cuestionada por docentes e investigadores relativamente desde hace poco tiempo: "la escritura académica universitaria, nunca ha sido objeto de preocupación constante e investigación en la educación superior y sólo hasta hace dos décadas en el contexto anglosajón, una década en el ámbito latinoamericano, y un lustro en Colombia, empezó a ser objeto de investigación sistemática y de enseñanza, por diferentes instituciones y comunidades académicas" (Ortiz 2011).

En la revisión de materiales de las autoras (Carrasco, Encinas, Castro, López 2013, p. 349) se aportó información con respecto al análisis metodológico que ellas mismas realizaron de otros autores, uno de éstos, se basó en la producción de ensayos. Aquí, Castro y Sánchez (2013) tienen como fin centrar su reflexión en los procesos de elaboración atendiendo no solo 
a la escritura académica sino a recursos con soportes digitales para la producción académica superior.

Otro material fue de Ávila, Gonzales y Peñaloza (2013) donde se aborda el cambio constitucional para la formación de lectores y escritores universitarios, en los cuales confirmaron la escasez de propuestas pedagógicas que faciliten la lectura de textos científicos, así como planteamientos para formar la escritura en este nivel.

Un caso más se refiere al análisis de producción textual de Miras, Solé y Castell (2013), maestras psicólogas de Barcelona. Ellas observaron síntesis escritas. De los resultados de aprendizaje arrojados por los alumnos, se dedujo que quienes tienen más creencias transaccionales, es decir, el uso integrado de relaciones cognitivas involucradas en la lectura y escritura, donde la combinación de ambas proporciona alternativas entre pensamiento y aprendizaje; muestran mejor organización e integración de contenidos, de tal manera que quienes elaboran textos más integrados obtienen mayores beneficios de aprendizaje.

\section{MÉTODO}

Para llevar a cabo esta investigación se recurrió a un solo grupo de primer semestre, 1H, de la materia de Lectura Expresión Oral y Escrita I, impartida por el maestro René Salas Zazueta; con un total de 40 alumnos. Se seleccionó al azar una muestra de 10 estudiantes con un promedio regular, mínimo de 80 . El análisis se basó en la producción de dos textos, siendo el primero un escrito diagnóstico, donde el alumno plasmó su propio conocimiento acerca del tema, consideró todos los aspectos que involucra el lenguaje escrito, es decir, la estructura léxico-morfológica-sintáctica que posee. Se proporcionó una instrucción general para la elaboración del mismo. El segundo fue un texto más complejo en donde los estudiantes no solo consideraron su acervo lingüístico, sino también el aprendizaje adquirido durante el curso de la materia, enfatizando el uso de coherencia y cohesión; es decir, la estructura correcta de diferentes tipos de párrafos así como su relación con los diferentes tipos de conectivos. 
Tabla de población y muestra por sexo

\begin{tabular}{|c|c|c|c|}
\hline $\begin{array}{l}\text { Total de la población } \\
\text { (Alumnos ) }\end{array}$ & $\begin{array}{l}\text { Total de la muestra } \\
\text { analizada }\end{array}$ & Sexo masculino & Sexo femenino \\
\hline $\mathbf{4 0}$ & $\mathbf{1 0}$ & $\mathbf{3}$ & $\mathbf{7}$ \\
\hline
\end{tabular}

\section{Instrumentos de recolección y análisis de datos}

Como se mencionó, la investigación es de tipo cuantitativo, específicamente es un estudio de caso inductivo de investigación-acción. Se realizó una interacción e introspección grupal, utilizando la observación, anotación, análisis e interpretación de textos en el contexto escolar.

Toda medición o instrumento de recolección de datos debe reunir tres requisitos esenciales: confiabilidad, validez y objetividad. El primero, se refiere al grado en que un instrumento produce resultados consistentes y coherentes; el segundo, al grado en que un instrumento coteja la variable que se busca medir; y el tercero, al grado en que el instrumento es permeable a la influencia de tendencias de los investigadores que administran, califican e interpretan (Hernández 2006).

Para el desarrollo del proyecto se elaboraron dos instrumentos de medición. El primero aludió a la transcripción original del texto de cada uno de los alumnos elegidos para el estudio; el segundo consideró las categorías que se analizaron en cada estructura proposicional de acuerdo al acervo lingüístico de cada uno de los participantes.

\section{Definición de categorías de análisis}

En este apartado se alude a las categorías de estudio que se consideraron para el análisis de información textual; entre éstas se encuentran en primer lugar, el mensaje, el cual es el contenido de la información que se emite sea oral o escrita. Forma parte de los componentes del circuito del habla; es el fin que se pretende alcanzar en cualquier conversación. En segundo lugar se considera tanto la idea principal como la idea secundaria: La principal, expresa la 
información básica para el desarrollo del tema que se trata; las secundarias abordan aspectos derivados del tema principal; sirven para ampliar, ejemplificar, aclarar. En tercer lugar está la coherencia, que es la propiedad de los textos de seleccionar y organizar la información para ser percibida de una manera clara y precisa por el receptor; está relacionada con la organización de información y con el conocimiento que comparten el emisor y receptor sobre el contexto. Para que se establezca con claridad se debe de considerar que todos los enunciados aborden un mismo tema; que todas las partes se encuentren organizadas entre sí (organización y estructuración interna); presentar una progresión temática de diversas formas y el emisor debe de tener el conocimiento del receptor sobre tema. La cuarta categoría es la cohesión, propiedad de los textos que consiste en la relación gramatical y semántica entre los enunciados escritos. Los mecanismos que la presentan son: De recurrencia, de sustitución, marcadores textuales y conectores textuales. Los primeros, consisten en la repetición de palabras, grupos de palabras u oraciones; los segundos, evitan la repetición de palabras, grupos de palabras u oraciones; los terceros, ayudan al receptor a interpretar el sentido del mensaje; los últimos, establecen relaciones entre las distintas partes del texto, algunos son de oposición, sumativos, de causaconsecuencia, de orden, etc. En quinto lugar, se considera a la puntuación, la cual indica una pausa en la estructura oracional así como el modo en que debe ser entendida. Ayuda a que todo lo redactado pueda ser leído y comprendido de forma clara y precisa. Entre los signos más utilizados encontramos: la coma, el punto, el punto y coma, signos de interrogación y exclamación, los dos puntos. En el sexto sitio se ubica el léxico: es decir, todas aquellas palabras que forman parte de una lengua (vocabulario). Constantemente se encuentra renovándose con nuevos términos, puede cambiar rápido o lentamente de acuerdo al uso y contexto. En séptimo lugar están los conectores: Se refiere a la palabra que une parte de un mensaje y establece una relación lógica entre oraciones; permite la conexión de enunciados en un texto. Pueden ser palabras, oraciones o conjunto de oraciones. Podemos encontrar diferentes tipos de conectivos: Copulativos, estos, sirven para añadir más información de la otorgada; disyuntivos, permiten establecer una separación, una opción; causales, muestran una causa; concesivos, establecen alguna opción o conceden algo imparcialmente; temporales, indican un momento en el tiempo; adición, suman, añaden información; locativos, hacen referencia a lugares; repetitivos, continuativos o de secuencia, señalan continuidad en las ideas; 
comparativos, indican comparación entre dos objetos; de consecuencia, un enunciado expresa la consecuencia de otro; condicionales, relaciona dos hechos, de manera que la realización de uno supone la realización del otro. Por último, están los aspectos gráficos, los cuales refieren a todos aquellos que se utilizan al momento de plasmar cualquier tipo de proposición, es decir, tipo de letra, trazo, legibilidad, ortografía, acentuación. En la tabla siguiente se presentan las categorías anteriores:

\begin{tabular}{|l|l|}
\hline \multicolumn{1}{|c|}{ Categorías de análisis } & \multicolumn{1}{c|}{ Datos } \\
\hline Coherencia & Coherencia de la idea principal con la idea secundaria \\
\hline Cohesión & Código o forma \\
\hline \multirow{2}{*}{ Estructura del párrafo } & Puntuación \\
\cline { 2 - 2 } & Sintaxis \\
\cline { 2 - 2 } & Conectores \\
\hline Léxico & \\
\hline Aspectos gráficos & \\
\hline
\end{tabular}

\section{ESTRATEGIA UTILIZADA EN LA PRODUCCIÓN TEXTUAL}

La estrategia utilizada por el maestro René Salas Zazueta en el Centro de Bachillerato Tecnológico Industrial y de servicio No. 11 (CBTis 11) para la enseñanza de tipología de párrafos, se basó principalmente en la propuesta de María Teresa Serafini quien parte primeramente del reconocimiento de las etapas que se manejan dentro de la escritura: preescritura y escritura. La primera, consiste en la selección de una idea a tratar, ideas de apoyo, cuál de éstas será la principal, cuáles la apoyarán, la desarrollarán, el establecimiento de orden y uso de conectores lógicos; la segunda, la redacción del párrafo y su revisión. Tal como lo propone Oviedo (1999): "Producción de un párrafo: preescritura: Selección idea nuclear o temática, acopio (lluvia) de ideas de apoyo, mapa conceptual (opcional), decisión estratégica (idea nuclear explícita e implícita; desarrollo (deductivo-inductivo), ordenamiento de las ideas, 
precisión de conexiones lógicas. Escritura: Redacción del párrafo (estrategias de escritura), revisión múltiple (reescritura o pulimiento).”

Tal propuesta avala lo establecido por el instrumento de registro de estrategias didácticas de la Subsecretaria de Educación Media Superior, cuyo propósito es el desarrollo de habilidades que permitan al estudiante comunicar en forma oral y escrita lo comprendido. Dentro de la comunicación escrita se aborda la cohesión y coherencia en párrafos, conectores distributivos y continuativos, idea principal, idea secundaria, oración simple y compuesta, donde se redactarán párrafos con diferentes estructuras lógicas y coherencia. Estos serán de tipo enumerativo, desarrollo de conceptos, comparativos, introductorios, cierre, causa-efecto, problema-solución; al redactarlos se considerarán: idea principal, puntuación y tipos de conectores dependiendo del tipo de párrafo que se desea plasmar.

Para su desarrollo se remitió a diferentes actividades, desde un escrito libre hasta dirigidos, en los cuales los alumnos siguieron procedimientos para su producción como la búsqueda de ciertas definiciones de términos, diversos textos así como análisis para la redacción de los mismos. También se presentó la transversalidad con documentos de otras materias que sirvieron de apoyo al estudiante.

La metodología que utilizó el maestro en esta institución es la siguiente: "La estrategia consiste en que el alumno escriba párrafos continuamente, por un lado, con cada una de las estructuras que ofrece Serafini; por otro, movilizando la lectura y los contenidos de las competencias disciplinares correspondientes. Para potenciar su competencia metalingüística, los alumnos escribían con tinta roja los conectores y la puntuación; asimismo, hacían explícita la estructura del párrafo al margen; también contaban palabras de cada oración principal o secundaria (el criterio para una oración era de +/- 20 palabras, y para un párrafo, de 60 a 120 palabras).

En la primera unidad, se escriben párrafos enumerativos con los contenidos de la temática de comunicación -comunicación verbal y no verbal, funciones de la lengua, etc.- contemplados en el programa para esa unidad; se enfatizó el posicionamiento de la idea principal y que presenta la enumeración, el uso de puntuación (dos puntos y punto y coma), y el uso de conectores de orden. 
En la segunda unidad se ejercita el párrafo desarrollo de concepto: en este párrafo se enfatiza la coherencia de la idea principal con las secundarias, el uso de conector de continuidad, y -lo más importante-, formas de argumentación en las ideas secundarias (ejemplos, citas, opiniones, datos estadísticos, etc.). Los contenidos disciplinares son lectura de textos literarios y párrafos con comentarios críticos y argumentos metalingüísticamente conscientes por parte del alumno. Otro párrafo es el comparativo, en el que se enfocan habilidades de análisis. Se parte de la definición cartesiana de análisis; luego se realizan análisis de dos objetos para compararlos; finalmente se ejercita la forma de redacción que propone Serafini (1996) para el párrafo comparativo. Se utilizan los conectores de oposición y se recuerdan los de orden. Se enfatiza una puntuación avanzada con puntos y comas y puntos y seguido. También se ve el párrafo de secuencia y se acentúa la diferenciación lógica entre enumeración y secuencia.

En la tercera unidad se leen capítulos de los libros de la Colección Ciencia para todos de Fondo de Cultura Económica sino con reconstrucción sintáctica, y exige comprensión del texto- de ocho párrafos. Se ven las últimas estructuras: causa efecto, con los conectores de causalidad; problema/solución, y los de introducción y conclusión.” (Salas 2014).

El tener conocimiento y seguir este tipo de estructura proposicional llevó al alumno a redactar textos con coherencia y cohesión, escribir un párrafo con ideas principales y secundarias; un ensayo con párrafos argumentados, ejemplos, datos, citas, y el uso de una puntación correcta que permitió separar las ideas secundarias de la principal. Los alumnos lograron redactar de manera clara, precisa y ordenada, además de mostrar una comprensión objetiva en la lectura de diversos textos. La implementación de la estrategia se realizó en su primera etapa, durante el primer periodo semestral del ciclo escolar 2013-2014, a partir de septiembre de 2013.

La propuesta del maestro Salas es la siguiente: "Que se enfoque la enseñanza de la escritura a partir de estructuras lógicas de párrafos y de formas de escritura de textos que reflejen la comprensión de la lectura de referencia (resumen, reseña crítica, ensayo). Para ello, 
que se diseñen textos que contribuyan a la profundización de la competencia metalingüística: esta es una forma de desarrollo cognitivo y de la capacidad lectora" (2014).

\section{RESULTADOS DE ANÁLISIS DE LAS CATEGORÍAS}

Coherencia. Respecto a esta categoría gramatical, todos los alumnos estudiados parten de una idea principal y la sustentan estructurando ideas secundarias; se mantienen en una coherencia básica, elemental. En algunos se presenta la ausencia de esta categoría.

Cohesión. La mayoría de los alumnos carecen del conocimiento, uso o ausencia de conectivos en sus estructuras oracionales; por lo tanto, el sentido que le otorgan a las proposiciones es incompleto.

\section{Estructura del párrafo:}

Puntuación. Casi todos los alumnos tienen los conocimientos mínimos en el uso de signos de puntuación; utilizan el punto, la coma, el punto y coma y los dos puntos como signos básicos. Sólo un estudiante presentó un nivel medio en su uso; otro, la usencia de punto final, otro más, la confusión entre el uso de coma y punto seguido.

Sintaxis. Los alumnos, en su mayoría, estructuraron oraciones coordinadas, subordinadas y yuxtapuestas. Redactaron el párrafo de desarrollo de concepto y algunos intentaron elaborar de tipo enumerativo; sin embargo, éste carece de conectores. La cantidad de palabras que utilizaron en cada uno varía. Por ejemplo, en el párrafo número uno, la cantidad mínima que se utilizó fue de 14 y la máxima de 22. En el párrafo número dos, los vocablos utilizados fueron 16 mínimo y 84 máximo. Cabe mencionar que para el desarrollo del escrito el maestro dio instrucciones, sin embargo, se presentaron dos casos donde los alumnos elaboraron tres párrafos, en los cuales, la cantidad de palabras utilizadas fueron 18 y 81 . Dentro de esta categoría se presentaron dos situaciones: En primer lugar la incoherencia, ésta marcada por el número, en la cual, el alumno escribió el verbo en singular cuando debería estar en plural; en segundo lugar, las repeticiones de términos o frases de algunos estudiantes.

Conectores. El uso de conectivos de coordinación, subordinación, yuxtaposición, desarrollo de concepto, son frecuentes en los estudiantes del grupo estudiado. Solo un alumno 
utilizó disyunción. Se intentó el uso desarrollo de concepto. No se usaron de causa-efecto, problema-solución, secuencial, comparativo.

Léxico. Al analizar la categoría perteneciente al acervo lingüístico, se dedujo que casi todos los alumnos poseen un nivel estándar; únicamente un alumno se acercó al culto, otro, al coloquial. Cabe señalar que en algunos casos la repetición es frecuente, ya sea en vocablos o frases.

Aspectos gráficos. En esta categoría, los alumnos presentaron diversidad en sus escritos; en la mayoría su letra fue legible aunque su trazo podría mejorar; cuidaron el uso de ortografía, mayúscula, aunque no en todos. Carecen de conocimientos de reglas de acentuación y de uso de signos de puntuación, cabe aclarar que no se presentó en todos. Tres estudiantes utilizaron título antes de comenzar su escrito.

Segunda fase: Resultados del texto final

En esta sección se abordó el análisis de textos de escritura final en alumnos del grupo 1H. Éste se redactó a partir de las instrucciones dadas por el docente. El escrito partió de varias lecturas que se realizaron a lo largo del semestre, por lo que debería mostrar los ocho párrafos estudiados y ejercitados: Introductorio, desarrollo de idea o concepto, de secuencia, enumerativo, comparación, causa-efecto, problema-solución y cierre y conclusión.

Primeramente se registró un acopio de ideas del tema; posteriormente se organizó el orden y la estructura de párrafo (tomando la lista de cotejo de cada uno de estos); después se elaboró un borrador y por último se presentó el texto en limpio. A continuación se muestra la Tabla y el escrito de transcripción textual que se utilizó en el análisis de cada alumno.

\section{TEXTO 2 ALUMNO:}

INSTRUCCIONES: Después de leer los capítulos sobre libros de la COLECCIÓN CIENCIA PARA TODOS escribe un texto de OCHO PÁRRAFOS en el que hables de los aprendizajes en LECTURA Y REDACCIÓN.

1.- Realiza los siguientes pasos del proceso de redacción: primeramente registra un acopio de ideas del tema a exponer; en segundo lugar, organiza en orden de la 
exposición y la estructura de párrafos; escribe un borrador; y por último, haz el texto en limpio.

2.- Moviliza la estructura de los párrafos estudiados: Introducción, desarrollo de idea o concepto, secuencia, enumeración, comparación, causa-efecto, problema-solución y cierre o conclusión (en cualquier orden, excepto el introductorio y el de conclusión, que van respectivamente al inicio y al final). Toma en cuenta la LISTA DE COTEJO para el tipo de párrafo.

\section{ACOPIO DE IDEAS (1-10)}

\section{ORGANIZACIÓN DEL TEXTO}

\begin{tabular}{|l|l|l|}
\hline Tipo de párrafo & $\begin{array}{l}\text { Orden } \\
\text { probable }\end{array}$ & Contenido del párrafo \\
\hline Introducción & & \\
\hline $\begin{array}{l}\text { Desarrollo de idea o } \\
\text { concepto }\end{array}$ & & \\
\hline Secuencia & & \\
\hline Enumeración & & \\
\hline Comparación & & \\
\hline Causa- efecto & & \\
\hline Cierre o conclusión & & \\
\hline
\end{tabular}

\section{ANÁLISIS Y DISCUSIÓN DE RESULTADOS}

Se analizaron dos escritos elaborados por alumnos de primer semestre del grupo $1 \mathrm{H}$, del CBTis No. 11. El primero, fue un diagnóstico de escritura libre que se redactó a partir de las instrucciones dadas por el docente. El texto cotejó tanto la competencia lingüística como la redacción del alumno. Dentro de la estructura proposicional se consideró la léxicomorfosintáctica de cada uno de los sujetos de estudio; enfocándose más en la coherencia y cohesión oracional así como en el tipo de conectores dentro de cada estructura. El segundo, se 
redactó también a partir de instrucciones dadas por el docente. Además que incluyó la estructura proposicional descrita anteriormente, también partió de varias lecturas realizadas a lo largo del semestre, contuvo los ocho párrafos estudiados y ejercitados: Introductorio, desarrollo de idea o concepto, de secuencia, enumerativo, comparación, causa -efecto, problema- solución, cierre y conclusión. Como primer paso se registró un acopio de ideas del tema; posteriormente se organizó el orden y la estructura de párrafo (tomando la lista de cotejo de cada uno de estos); después se elaboró un borrador y por último se redactó el texto en limpio.

El análisis de resultados fue arrojado a través de las categorías propuestas. La primera se refiere a la coherencia; en el escrito diagnóstico los alumnos asumieron elaborar tanto oraciones simples como compuestas, sin embargo, no fue así, ya que no cumplen con la cantidad de palabras que debe contener una proposición para que se considere como tal $(+/-$ 20 palabras). El escrito final muestra lo contrario, todos los alumnos estudiados partieron de una idea principal más estructurada y la sustentaron con ideas secundarias complejas. El número de vocablos que utilizaron aumentó, es decir los párrafos contaron con la cantidad de palabras indicadas para su estructura (60-120 palabras).

La segunda categoría de estudio abordó la cohesión. En el diagnóstico, la mayoría de los alumnos carecieron del conocimiento, uso o ausencia de conectivos en sus estructuras oracionales, por lo tanto, el sentido que le otorgaron a las proposiciones fue incompleto. En el texto final, utilizaron conectivos y signos de puntuación de manera adecuada, a su vez, se presentó un aumento en el uso de diversidad de estos.

La tercera categoría, la estructura de párrafo, manejó las siguientes subcategorías: Puntuación, en el escrito diagnóstico, los estudiantes arrojaron un conocimiento mínimo en el uso de signos de puntuación; utilizaron el punto, la coma, el punto y coma y los dos puntos como signos básicos. Sólo uno presentó un nivel medio; otro, la usencia de punto final, otro más, la confusión entre el uso de coma y punto seguido. En el escrito final, la mayoría mostró más conocimiento utilizaron el punto, la coma, el punto y coma, los dos puntos, las comillas de manera adecuada. El manejo de cada uno de estos se observó con más cuidado y en un contexto acorde; además, fue consciente, ya que al momento de plasmarlos se fijó con tinta roja. La segunda subcategoría fue la sintaxis. En el diagnóstico, los estudiantes asumieron que 
elaboraron proposiciones, sin embargo, la cantidad de palabras que utilizaron no correspondían al indicado; lo mismo sucedió con la estructura de párrafos. En el texto final, la mayoría, estructuró oraciones coordinadas, subordinadas y yuxtapuestas, además de que lograron redactar los ocho tipos de párrafo: desarrollo de concepto, enumerativo, secuencia, causa-efecto, problema-solución, introductorio, cierre, comparativo. La cantidad de palabras que utilizaron en cada uno varió. Por ejemplo, en el párrafo número uno, la cantidad mínima que se utilizó fue de 14 y la máxima de 22. En el número dos, los vocablos utilizados fueron 16 mínimo y 84 máximo. Cabe mencionar que para el desarrollo del escrito el maestro dio instrucciones, sin embargo, se presentaron dos casos donde los alumnos elaboraron tres párrafos, en los cuales la cantidad de palabras utilizadas fueron 18 y 81. Dentro de esta categoría se suscitaron dos situaciones más: La incoherencia, ésta marcada por el número, es decir, un alumno escribió el verbo en singular y debería estar en plural; así como las repeticiones de términos o frases en los escritos de algunos estudiantes. La tercer subcategoría hace referencia al uso de conectores. En el diagnóstico, éste se plasmó sin el conocimiento adecuado; es decir, se elaboran oraciones de tipo coordinante y subordinante; sin embargo, no se consideraron como tal por la cantidad de vocablos utilizados, en algunos casos estaban descontextualizados. En el texto final, el uso de conectivos de coordinación, subordinación, yuxtaposición, así como de los ocho tipos de párrafos que fueron frecuentes, se plasmaron de manera consciente ya que, se escribieron con tinta roja y en el contexto indicado. La cuarta subcategoría se refiere al léxico; en el diagnóstico, se dedujo que casi todos los alumnos poseyeron un nivel estándar; únicamente un alumno se acercó al culto, otro, al coloquial. Cabe señalar que en algunos casos la repetición fue constante, ya sea en vocablos o frases. En el final, la categoría perteneciente al acervo lingüístico, arrojó que casi todos poseen un nivel estándar más avanzado, ya que se percibió el aumento de vocablo. La repetición es menos frecuente. La quinta y última subcategoría aborda los aspectos gráficos. En el diagnóstico los alumnos presentaron diversidad en sus escritos; en la mayoría su letra fue legible aunque su trazo podría mejorar; cuidaron el uso de ortografía, de mayúscula, aunque no en todos los casos. Carecieron de conocimientos de reglas de acentuación y de uso de signos de puntuación, esta situación no se presentó en todos. Tres estudiantes utilizaron título antes de comenzar su texto. En el escrito final, casi todos arrojaron letras legibles, su trazo presentó una mejoría; la 
mayoría cuidó el uso de ortografía sin embargo, sí se presentó confusión en el uso de ciertas grafías como: /s/, /c/,/z/, /x/, /b/, /v/, /y/, /ll/. El uso inadecuado de mayúscula se mostró solo en un estudiante, el cual no la utilizó después de un punto y seguido. Algunos carecieron de conocimientos de reglas de acentuación, esto no fue general; sin embargo, hay situaciones en las cuales se no acentuó ningún término.

Es importante señalar que la carencia de algunos aspectos de redacción encontrados en el análisis de los escritos antes mencionados no intervienen en el desarrollo de la estrategia de enseñanza de estructura de párrafos; ya que, el propósito se logró, los alumnos al final del semestre elaboraron proposiciones con una competencia alta, escribieron párrafos complejos, estructurados, utilizaron puntuación adecuada, así como conectores que reflejan la lógica del discurso; se puede sustentar el desarrollo y ampliación de ideas en los estudiantes.

\section{CONCLUSIONES}

En la etapa diagnóstica se obtuvieron indicadores sobre la estructura oracional como escasa y deficiente; los alumnos no lograron diferenciar el componente de una oración, cuántas palabras deben estructurarla, mucho menos identificar un párrafo, cuantas oraciones lo componen. Tampoco reconocieron el uso de cada conectivo y tipo de párrafo. Sin embargo, es importante decir, que lograron redactar oraciones coordinantes, subordinantes y en ocasiones yuxtapuestas. Las proposiciones que elaboraron con más frecuencia fueron simples; la cantidad máxima de párrafos que redactaron fueron tres.

Conforme avanzó el curso, los estudiantes aprendieron, elaboraron y corrigieron oraciones así como párrafos; llegando a producir un texto final completo y adecuado al contexto. En éste, reconocieron el uso de conectivos así como de los ocho tipos de párrafos basados en la propuesta de Serafini: Introductorio, desarrollo de concepto, enumerativo, de secuencia, de problema-solución, comparativo, causa-efecto, cierre. Es importante enfatizar que en este nivel los alumnos no solo estructuraron oraciones simples sino también complejas; a su vez llegaron a producir los ocho tipos de párrafos.

Analizados estos dos momentos -el diagnóstico y la aplicación de la estrategia- se observó una diferencia distante entre ambos. La metodología empleada para la enseñanza de la 
escritura en la institución de estudio (CBTis 11) permitió que los alumnos realmente lograran redactar escritos complejos con coherencia y cohesión. Estos fueron precisos, entendibles y extensos; cuidaron su acervo lingǘstico así como otros aspectos de nuestra escritura, tal como el uso correcto de signos de puntuación, de diversos tipos de conectivos y la estructura proposicional.

Conviene mencionar, como una recomendación técnico-pedagógica, que la propuesta de enseñanza utilizada debería ser implementada en este nivel educativo, primeramente, por todos los maestros del área de estudio en la propia escuela, posteriormente, en las otras instituciones educativas del estado. (Ver anexos de ejemplos de escrito diagnóstico (texto 1) y escrito final (texto 2).

\section{REFERENCIAS BIBLIOGRÁFICAS}

Ávila, Natalia; Paula González y Christian Peñaloza. "Creación de un programa de escritura de una Universidad Chilena: Estrategias para promover un cambio institucional”. Revista Mexicana de Investigación Educativa. 18.57. (2013): 537-560.

Cáceres, Orlando. “¿Qué es la gramática? Ortografía y redacción”. About.com. 2008. http://reglasespanol.about.com/od/reglasgramaticales/f/Qu-E-Es-La-GramAtica.htm

Carrasco, Alma, María Cristina Castro y Guadalupe López. Lectura y escritura académica en la educación media superior y superior. Revista Mexicana de Investigación Educativa. 18.57. (2013): 349-354.

Castro, María Cristina y Martín Sánchez. "La expresión de opinión en textos académicos escritos por estudiantes universitarios”. Revista Mexicana de Investigación Educativa. 18.57. (2013): 483-506.

Gili Gaya, Samuel. Curso superior de sintaxis del español. Barcelona: Vox, 1993.

Gómez Palacio, Margarita. La producción de textos en la escuela. México: SEP. 1997.

Hernández, Roberto, Carlos Fernández y Pilar Baptista. Metodología de la Investigación. 4ta. Edición. México: McGrawHill. 2006. 
Miras, Mariana e Isabel Solé. “Creencias sobre la lectura y la escritura, producción de síntesis escritas y resultados de aprendizaje". Revista Mexicana de Investigación Educativa. 18.57. (2013): 437-459.

Real Academia Española. Nueva gramática de la lengua española, manual. España: Espasa libros. 2009.

Rodríguez, Ada Nelly. Lectura crítica y escritura significativa: Acercamiento didáctico desde la Lingüistica. Laurus. 13.25. (2007): 241-262.

Serafini, María Teresa. Cómo se escribe. Madrid: Paidós. 1996.

Subsecretaría de Educación Media Superior. Instrumento de estrategias didácticas. Sonora: DGETI. 2013.

Toledo, Alicia; María Teresa Godoy y Zaida Suárez. "El análisis semántico, sintáctico y pragmático en la enseñanza de los contenidos gramaticales”. VARONA, Revista Cientifico-Metodológica, No. 46. (2008): 60-65.

\section{ANEXOS}

\section{ANEXO 1}

A continuación se presentan dos escritos producidos por los alumnos del CBTis 11, en estos se respetó la transcripción original de los mismos.

TEXTO 1 ALUMNO 1: Ríos Pérez Verónica Lizeth.

INSTRUCCIONES: Escribe dos párrafos con estructura, mecanismos de coherencia y con base en norma lingüística. 


\section{Tema: La computadora}

La computadora es un aparato donde podemos procesar y guardar datos, documentos, fotos, etc.

La computadora nos sirve para investigar las cosas que no sabemos y nos llaman la atención saber, por ejemplo un libro, un pintor, un autor, un lugar y todas esas cosas que nos llaman la atención. También nos sirven para hacer tareas en algún programa o simplemente investigar. Para entrar a redes sociales como facebook, twitter, para hablar con amigos, familia, etc.

\section{TEXTO 1 ALUMNO 2: Fernanda Amaya Aguilar.}

INSTRUCCIONES: Escribe dos párrafos con estructura, mecanismos de coherencia y con base en norma lingüística.

\section{Tema: La computadora}

La computadora es una máquina que nos sirve para procesar datos y así mismo convertirlos en información útil.

Está compuesta por el CPU, un monitor, teclado, mouse y bocinas; estos son sus componentes físicos.

Sus componentes interiores lo forman el software, hardware, dispositivos de entrada y salida, entre otros.

\section{ANEXO 2}

\begin{tabular}{|c|l|l|}
\hline $\begin{array}{c}\text { Categorías de } \\
\text { Análisis }\end{array}$ & Datos & \multicolumn{1}{|c|}{$\begin{array}{c}\text { Alumno: 1 } \\
\text { Características Diagnóstico }\end{array}$} \\
\hline Coherencia & $\begin{array}{l}\text { Coherencia de la } \\
\text { idea principal con } \\
\text { la idea secundaria }\end{array}$ & $\begin{array}{l}\text { Emplea una idea principal, amplía la información } \\
\text { utilizando ideas secundarias. La coherencia es básica. }\end{array}$ \\
\hline
\end{tabular}




\begin{tabular}{|c|c|c|c|c|}
\hline Cohesión & Código o Forma & $\begin{array}{l}\text { El alumno prese } \\
\text { oracional, ya que } \\
\text { tema a desarrolla } \\
\text { uso adecuado de }\end{array}$ & $\begin{array}{l}\text { cohere } \\
\text { e manti } \\
\text { En cuar } \\
\text { nectore }\end{array}$ & $\begin{array}{l}\text { ásica en la estructura } \\
\text { el contexto sobre el } \\
\text { a cohesión carece del } \\
\text { darle un sentido más }\end{array}$ \\
\hline \multirow{3}{*}{$\begin{array}{c}\text { Estructura del } \\
\text { Párrafo }\end{array}$} & Puntuación & $\begin{array}{l}\text { Mínima } \\
\text { Sólo utiliza } \\
\text { comas, punto, } \\
\text { punto y seguido. }\end{array}$ & Media & Avanzada \\
\hline & Sintaxis & \multicolumn{3}{|c|}{$\begin{array}{l}\text { Utiliza oraciones coordinadas conjuntivas y disyuntivas } \\
\text { así como yuxtapuestas. Desarrollo de párrafo de } \\
\text { concepto. En el párrafo } 1 \text { utiliza } 14 \text { palabras, en el } \\
\text { segundo. } 63 \text {. }\end{array}$} \\
\hline & Conectores & \multicolumn{2}{|c|}{$\begin{array}{l}\text { Si utiliza } \\
\text { Disyuntivos } \\
\text { Coordinante } \\
\text { Desarrollo de concepto }\end{array}$} & No utiliza \\
\hline
\end{tabular}

1. TABLAS DE CATEGORÍAS DE ANÁLISIS

\begin{tabular}{|c|c|c|c|}
\hline Léxico & Coloquial & $\begin{array}{l}\text { Estándar } \\
\text { Maneja un léxico de este tipo, } \\
\text { es repetitivo. }\end{array}$ & Culto \\
\hline $\begin{array}{l}\text { Aspectos } \\
\text { gráficos }\end{array}$ & \multicolumn{3}{|c|}{$\begin{array}{l}\text { La letra es legible, aunque podría tener un mejor trazo; cuida la ortografía, } \\
\text { carece de conocimiento sobre las reglas de acentuación. No cuida el uso de } \\
\text { mayúsculas. }\end{array}$} \\
\hline
\end{tabular}

\begin{tabular}{|l|l|l|}
\hline $\begin{array}{c}\text { Categorías } \\
\text { de Análisis }\end{array}$ & Datos & \multicolumn{1}{c|}{$\begin{array}{c}\text { Alumno: } 2 \\
\text { Características Diagnóstico }\end{array}$} \\
\hline Coherencia & $\begin{array}{l}\text { Coherencia de la } \\
\text { idea principal con } \\
\text { la idea secundaria }\end{array}$ & $\begin{array}{l}\text { Redacta una idea principal, amplía la información } \\
\text { con ideas secundarias, la coherencia es básica. }\end{array}$ \\
\hline Cohesión & Código o Forma & $\begin{array}{l}\text { Presenta una coherencia básica, elemental. Carece de } \\
\text { uso de conectores de manera adecuada. }\end{array}$ \\
\hline
\end{tabular}




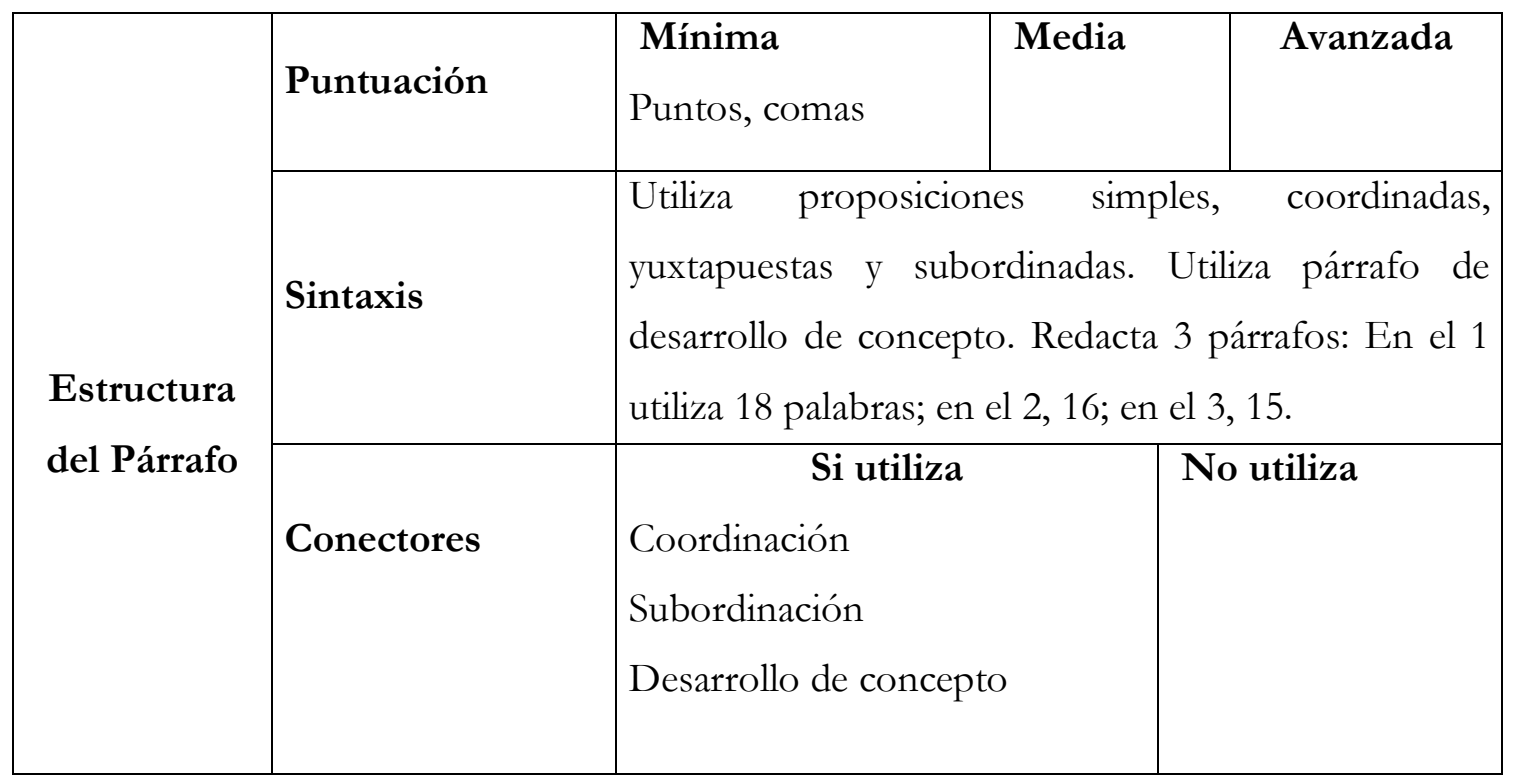

\begin{tabular}{|l|l|l|l|} 
Léxico & \multicolumn{1}{|c|}{ Coloquial } & \multicolumn{1}{|c|}{$\begin{array}{c}\text { Estándar } \\
\text { Maneja este tipo de léxico. }\end{array}$} & Culto \\
\hline $\begin{array}{l}\text { Aspectos } \\
\text { gráficos }\end{array}$ & $\begin{array}{l}\text { La letra es legible, aunque podría mejorar su trazo, respeta el uso de } \\
\text { mayúsculas, acentos y reglas de ortografía. }\end{array}$ \\
\hline
\end{tabular}

ANEXO 3 (Texto 2)

2. ORGANIZACIÓN DEL TEXTO

\begin{tabular}{|l|l|l|}
\hline \multicolumn{1}{|c|}{ Tipo de párrafo } & $\begin{array}{l}\text { Orden } \\
\text { probable }\end{array}$ & Contenido del párrafo \\
\hline Introducción & 6 & De que se trata. \\
\hline
\end{tabular}




\begin{tabular}{|l|c|l|}
\hline $\begin{array}{l}\text { Desarrollo de idea o } \\
\text { concepto }\end{array}$ & 2 & Porqué son importantes en un texto. \\
\hline Secuencia & 3 & $\begin{array}{l}\text { Cuáles son los pasos importantes de realizar } \\
\text { un buen texto. }\end{array}$ \\
\hline Enumeración & 4 & Cuál es la importancia de la escritura. \\
\hline Comparación & 1 & Diferencias entre la lectura y la escritura. \\
\hline Causa- efecto & 7 & Cuáles son los problemas al no saber \\
primeramente un texto.
\end{tabular}

\section{TEXTO 2 ALUMNO 1: Ríos Pérez Verónica Lizeth.}

(Párrafo introductorio)(6)

En estos párrafos vamos a hablar sobre la lectura y la escritura; cuales son los aprendizajes y conocimientos que nos dejan; como desarrollar un poco más sobre nuestro modo de escribir. También como nos ayuda como organizar nuestras ideas para la realización de un texto, las ventajas que tenemos y todos aquellos procesos que se llevan a cabo.

(Párrafo enumerativo) (4)

Sabemos que la escritura es esencial en nuestra vida; Primeramente, nos enseñan a escribir al pasar a la escuela o algunas veces nuestros padres nos enseñan; Después, mejoramos nuestro modo de escritura al ir creciendo pero no lo suficiente para saber cuál es su secuencia, porque como todo, tiene sus reglas; por último, para mejorar, una de las cosas que nos ayuda mucho, es leer.

(Párrafo de secuencia) (3)

Para poder o saber cómo llevar a cabo un texto, por eso aquí hay algunos pasos: 1.- La lectura: Cuenta mucho ya que, claro está, sin ella no podemos realizar el texto; 2.- Acopio de las ideas, 
para que se nos haga más fácil al desarrollar nuestro texto; 3.- Los conectos; que son aquellas puntuaciones claves que hacen ver a nuestro texto más formal y comprensible.

(Párrafo de desarrollo de idea o concepto) (2)

Los conectores son las "puntuaciones", como la coma, punto y coma, palabras, etc. Lo que son tan importantes en un texto. En efecto los conectores son tan importantes ya que ayuda al texto y a nosotros a diferenciar cuál es la idea principal, que sigue después de un punto y coma. Así bien, nos permite dar una mejor estructura a nuestro párrafo y así a su vez hayamos más maneras de cómo escribir mejor.

(Párrafo comparativo) (1)

La lectura y escritura son dos cosas diferentes, pero al fin de cuentas se relacionan: La lectura nos ayuda a desarrollar nuestro conocimiento y pensamiento, en cambio, la escritura nos ayuda al modo de realizar un cuento, o cualquier otro tipo de texto, sin embargo la lectura nos permite desarrollar nuestra forma de hablar, porque así descubrimos nuevas palabras, al final la escritura y la lectura son importantes en nosotros.

(Párrafo problema-solución) (5)

En la actualidad, tanto como niños, jóvenes y adultos están atrapados en la tecnología, y la usan para realizar cualquier investigación, que ya ni toman en cuenta un libro o cualquier otro medio. ¿Cómo podemos llegar a una solución? Recomendaría un libro que se titula "El ábaco, la liria y la rosa que habla un poco sobre estos problemas, pero el caso es que hay que dar consejos y recomendaciones.

(Párrafo causa-efecto) (7)

En consecuencia de mi grupo no sabía cómo realizar ningún tipo de párrafos o bien no tenías idea de lo que podías hacer, el maestro nos encargó leer un libro llamado "El hombre y los materiales" y de esa lectura nos encargó realizar 7 párrafos con su orden y sus conectores. El efecto que tuvo esta actividad fue que aprendimos a utilizar conectores y saber cómo recolectar nuestras ideas pero sobre todo adquirir conocimientos nuevos.

(Párrafo cierre o conclusión) (8)

Núñez doi: https://doi.org/10.36799/el.v111.25 Volumen 1, Número 1, Año 2016, ISSN: 2448-5942 
Mi conclusión al final de todo es que con la lectura y la escritura aprendimos la realización de párrafos. También adquirimos nuevos conocimientos al haber leído "El hombre y sus materiales", "la muerte y sus ventajas", "El ábaco, la liria y la rosa" y la de sección libre que yo escogí fue "La vida, el tiempo y la vejez". De todos estos adquirimos nuevos conocimientos.

TEXTO 2 ALUMNO 2: Amaya Aguilar Fernanda

INSTRUCCIONES: Después de leer los capítulos sobre libros de la COLECCIÓN CIENCIA PARA TODOS escribe un texto de OCHO PÁRRAFOS en el que hables de los aprendizajes en LECTURA Y REDACCIÓN.

1.- Realiza los siguientes pasos del proceso de redacción: primeramente registra un acopio de ideas del tema a exponer; en segundo lugar, organiza en orden de la exposición y la estructura de párrafos; escribe un borrador; y por último, haz el texto en limpio.

2.- Moviliza la estructura de los párrafos estudiados: Introducción, desarrollo de idea o concepto, secuencia, enumeración, comparación, causa-efecto, problema-solución y cierre o conclusión (en cualquier orden, excepto el introductorio y el de conclusión, que van respectivamente al inicio y al final). Toma en cuenta la LISTA DE COTEJO para el tipo de párrafo.

\section{ACOPIO DE IDEAS}

1.- Lectura

2.- Escritura

3.- Mencionar los libros

4.- Estructura de párrafos

5.- El problema de la lectura actualmente

Núñez doi: https://doi.org/10.36799/el.v1i1.25 Volumen 1, Número 1, Año 2016, ISSN: 2448-5942 
6.- Lectura y escritura

7.- La escritura de hoy en día y como era años atrás

8.- Conclusión

9.- La importancia de los libros

\section{ORGANIZACIÓN DEL TEXTO}

\begin{tabular}{|l|c|l|}
\hline Tipo de párrafo & Orden probable & Contenido del párrafo \\
\hline Introducción & 1 & $1,2,3$ \\
\hline $\begin{array}{l}\text { Desarrollo de idea o } \\
\text { concepto }\end{array}$ & 7 & Importancia de los libros \\
\hline Secuencia & 4 & Estructura de párrafos \\
\hline Enumeración & 2 & Mencionar los libros \\
\hline Comparación & 6 & $\begin{array}{l}\text { La escritura hoy en día y como era } \\
\text { antes }\end{array}$ \\
\hline Causa- efecto & 5 & Lectura y escritura \\
\hline Problema-solución & 3 & $\begin{array}{l}\text { El problema de la lectura } \\
\text { actualmente }\end{array}$ \\
\hline Cierre o conclusión & 8 & Conclusión \\
\hline
\end{tabular}

\section{Texto del alumno:}

Párrafo introductorio (1)

En este ámbito hablaré de todo lo que vimos en este 3er parcial. Me pareció bastante interesante todo lo que vimos ya que aprendimos a estructurar párrafos, a leer adecuadamente; cabe recalcar los cinco libros que leímos. En efecto, fue algo muy importante para practicar todo lo que fuimos aprendiendo en este mes.

Párrafo enumerativo (2) 
A lo largo del parcial leímos y aprendimos sobre los siguientes 5 libros: Primeramente, vimos el libro "El hombre y los materiales" que habla de la vida en la prehistoria; en segundo lugar, leímos sobre "Del acero a los polímeros" que trata de la evolución del hombre y los materiales; en tercer lugar, está el libro "El ábaco, la lira y la rosa" que habla más que nada sobre el arte; por último, el libro "La muerte y sus ventajas” que en lo personal fue el que más me gustó.

Párrafo problema-solución (3)

Saber leer es algo esencial para la vida. En efecto no solo es leer sino entender lo que estamos leyendo y aplicarlo. Hoy en día las generaciones no le toman tanta importancia a la lectura ¿Cómo podríamos resolver este problema? Una solución sería practicar más la lectura y no hacerlo por obligación sino por gusto.

Párrafo de secuencia (4)

Para realizar un buen párrafo se debe tener en cuenta lo siguiente:

1.- Primeramente, debemos de hacer una buena lectura del libro o texto y más que nada entenderlo.

2.- Ya leído el texto se debe tener muy claro que es lo que queremos plasmar en el párrafo.

3.- El siguiente paso es estructurarlo mentalmente.

4.- Por último, toca escribirlo y terminamos.

Párrafo causa-efecto (5)

La lectura y la escritura hoy en día sinceramente no es como quisiéramos. Esto se debe a que actualmente préstamos más importancia a otras cosas sin sentido; esto sucede más en los jóvenes ya que piensan que es aburrido y lo tratan de evitar como se pueda, lo que no sabemos es que en un futuro esto nos va a perjudicar mucho.

Párrafo comparativo (6) 
La escritura actualmente y la escritura años atrás es bastante diferente. Hoy en día estamos muy actualizados ya sea en escritura, idiomas, entre otros. Mientras que en el pasado con trabajo sabían el abecedario y no sabían hablar con coherencia, cabe recalcar que la escritura ha existido desde hace miles de años y es un factor importante para la comunicación.

Párrafo de desarrollo de idea (7)

Se aprende más en los libros que en otros medios. En efecto, los libros son algo que personalmente pienso que ayuda bastante para abrir la mente y las ideas. Ya que como solo estás leyendo te imaginas todo el contenido del libro enriqueces tu mente y te ayuda a ser más inteligente en el sentido de entender todo el contenido del libro.

Párrafo de conclusión (8)

En definitiva, saber escribir y saber leer me parece algo sumamente importante ya que influye mucho en la comunicación; en la actualidad, la mayoría de las personas nos comunicamos por vía internet ya sea por correos, e-mails, etc. Nadie pensó que estos dos factores que son la escritura y la lectura se convertirían en algo fundamental para vivir. 